Fortnightly Review

\title{
Drugs in sport
}

\section{Domhnall MacAuley}

The use of drugs to enhance performance in sport will not go away. Athletes seek every competitive advantage and the rewards of success at top level are great, both financially and in personal glory. Almost all top level competitors are full time and, even if not paid, are to all purposes professional. There is huge pressure to train longer and harder and to take a scientific approach to nutrition and fluid and electrolyte balance, to seek every biomechanical and psychological advantage. It is almost inevitable that some will seek an advantage through drugs. Though there may be little clear objective scientific evidence of a benefit to be gained from drug use, what evidence there is supports the widespread belief among athletes that drugs help. Indeed, many believe that it is impossible to succeed without drugs. Though an athlete's motivation in taking drugs is understandable, we cannot condone it. Firstly, it can be dangerous to the athlete's health and, secondly, it is against all principles of fair competition.

\section{Method}

A systematic search of the literature is unlikely to tell the complete story in any review of drug abuse in sport. Doping in sport is essentially an underground activity with little formal published research in a topic which should not officially exist. Much of what is common knowledge is cloaked in rumour, suspicion, and suggestion, and the strategies adopted by athletes seem to be founded more on empiricism than published scientific data.

The information presented in this review has been identified from several sources. A Medline search from 1966 to 1996 identified 620 references to the term "doping in sports." With the search restricted to humans (504) and to publications in English there were 389 references. Those references were subclassified into publications identifying side effects, prevalence, methods of detection, and a fourth group composed of editorials, opinions, and reviews. Further references were identified from a search of the National Sports Medicine Institute database and a hand search of the past five years of the British fournal of Sports Medicine. Additional information is available from the annual report of the Sports Council ${ }^{1}$ and advisory booklets from the same source. ${ }^{2-7}$

\section{Classes of banned substances}

Drugs prohibited by the International Olympic

British Journal of Sports Medicine, London

WC1H 9JR

Domhnall MacAuley, editor

Correspondence to: Hillhead Family Practice, Belfast BT11 9FZ.
Committee include those listed below (see box).

\section{STIMULANTS}

Amphetamines are used by athletes to increase aggression and competitiveness and to reduce tiredness and fatigue and have a long history of abuse, particularly in cycling. Adverse medical side effects may include a rise in blood pressure and body temperature, arrhythmias, aggression, anxiety, and addiction.

\section{International Olympic Committee Medical Commission}

Prohibited classes and substances and prohibited methods

Doping contravenes the ethics of both sport and medical science. Doping consists of the administration of substances belonging to prohibited classes of pharmacological agents or the use of various prohibited methods, or both

\section{Prohibited classes of substances}

- Stimulants

- Narcotics

- Anabolic agents

- Diuretics

- Peptide and glycoprotein hormones and analogues

Prohibited methods

- Blood doping

- Pharmacological, chemical, and physical manipulation

Classes of drugs subject to certain restrictions

- Alcohol

- Marijuana

- Local anaesthetics

- Corticosteroids

- $\beta$ Blockers

Caffeine $^{8}$ is slightly unusual owing to its widespread social use and presence in many beverages. There is a threshold level and the concentration of caffeine in the urine should not exceed $12 \mu \mathrm{g} / \mathrm{ml}$. One group that causes particular problems is the sympathomimetic amines, of which ephedrine, pseudoephedrine, phenylpropranolamine, and norpseudoephedrine are examples. These remain on the prohibited list, though doubts remain about their ergogenic effect.

\section{NARCOTIC ANALGESICS}

Narcotic analgesics reduce pain sensitivity and enable an athlete to continue despite injury. Adverse effects of cocaine abuse have been recorded in athletes. Recent changes in the International Olympic Committee doping regulations mean that codeine, dihydrocodeine, and pholcodine are now permitted analgesics; it should be noted, however, that dextropropoxyphene remains a prohibited substance.

\section{ANABOLIC STEROIDS}

Anabolic steroids are probably the best known drugs of abuse, though the evidence for their effectiveness had 
not been convincing' until the recent publication of a randomised controlled trial with high dose testosterone. ${ }^{10}$ Side effects are well documented. They include psychological and psychiatric conditions, rupture of the musculocutaneous junction, gynaecomastia, hypogonadism, effects on coagulation and lipids and lipoproteins, cholestasis, skin disease, hypertension, stroke, and myocardial infarction. Chemical structures have been modified to increase the anabolic effect and reduce the androgenic effect and more than 100 different anabolic steroids are available, taken either by mouth or by injection. Most often used for their anabolic or muscle building effect, they also affect mood and aggression, which enables people to train harder.

Classically anabolic steroids are taken by power athletes, so are widely used and abused by body builders and recreational weight trainers, but they are also reputedly used as a training aid by endurance athletes to improve recovery from training loads. Doses greatly exceed the normal therapeutic doses and athletes may take several different types of anabolic steroid together (stacking) or vary the use of different steroids (cycling). Because these drugs are used essentially as training aids athletes may stop some weeks before an event and later pass the competition dope test. Clearly, if athletes knew their own clearance time for a particular oral or injectable drug they could plan drug use to give the maximum benefit with least risk of detection. Other drugs used concurrently may include diuretics to reduce fluid retention, thyroxine to promote weight loss, and tamoxifen to prevent gynaecomastia. These agents are quite freely available in gymnasiums and fitness clubs throughout Britain.

Because of the difficulties in testing for testosterone the testosterone to epitestosterone ratio is used as the clue to detection. $\beta_{2}$ Agonists used systemically also have powerful anabolic effects, hence clenbuterol is banned. Other $\beta_{2}$ agonists are also prohibited, though salbutamol, salmeterol, and terbutaline, which are prohibited for systemic use, are permitted by inhalation if previously declared. Doubts remain about the possibility of false positive test results, ${ }^{11}$ and indeed a recent paper examined the effect on drug tests of eating meat from steroid treated livestock. ${ }^{12}$

\section{$\beta$ BLOCKERS}

$\beta$ Blockers are used both to control the effects of anxiety and, in some sports-notably shooting ${ }^{13}$ and archery - to produce bradycardia. In these sports and others in which accuracy and control are important, such as bowls, $\beta$ blockers have great potential effect; but they are clearly of little use in physically active sports. Other sports in which the use of $\beta$ blockers is banned include bobsleigh and luge, ski jumping and free style skiing, diving and synchronised swimming, and modern pentathlon.

\section{DIURETICS}

Diuretics have been abused in those sports in which athletes compete at weight limits and are used to shed weight quickly. They have also been used to increase urine volume and dilution to make detection of small quantities of banned substances more difficult.

\section{PEPTIDE HORMONES}

Peptide hormones, the so called sports designer drugs, may be used for several reasons. Their main attraction from the athlete's viewpoint is the difficulty of detection. Human growth hormone is used for its anabolic effect. The possibility that some growth hormone preparations of human origin may be associated with Creutzfeldt-Jacob disease has caused anxiety among some athletes. Corticotrophin increases the level of endogenous corticosteroids and may alter mood.
Human chorionic gonadotrophin is used to increase the production of endogenous steroids.

\section{“BLOOD DOPING"}

Athletes have always been aware of the possible benefit of improving oxygen carrying capacity in endurance sport, hence many train at altitude. More recently athletes have used blood doping - in which blood is taken off, stored, and later reinfused, thereby boosting the packed cell volume. Blood doping, which is effective, ${ }^{14}$ is banned but exceptionally difficult to detect. It does, however, carry all the logistical problems associated with storage and reinfusion in less than ideal conditions. Erythropoietin, initially developed to counter severe anaemia in renal failure, has been used by athletes as a more convenient method of increasing the packed cell volume. Its use is banned but currently undetectable. It is suspected but unproved that the sudden and unexplained death of some endurance athletes may be associated with the uncontrolled use of erythropoietin.

\section{MANIPULATION OF PROCEDURES AND OTHER DRUGS}

Pharmacological, chemical, and physical manipulation of the drug testing procedure is also prohibited. This includes physical methods such as catheterisation, urine substitution, and tampering with samples. It also includes methods to inhibit renal excretion - for example, by using probenecid and related substances. Using epitestosterone to correct the ratio to testosterone is also prohibited.

Certain other drugs are subject to restriction. They include alcohol, marijuana, and local anaesthetics. Certain local anaesthetics are permitted for local or intra-articular use, and then only when medically justified and with prior notification to the relevant medical authority. Corticosteroids are permitted for topical use only (by inhalation and intra-articular and local injection), and then only with written notification to the relevant medical authority.

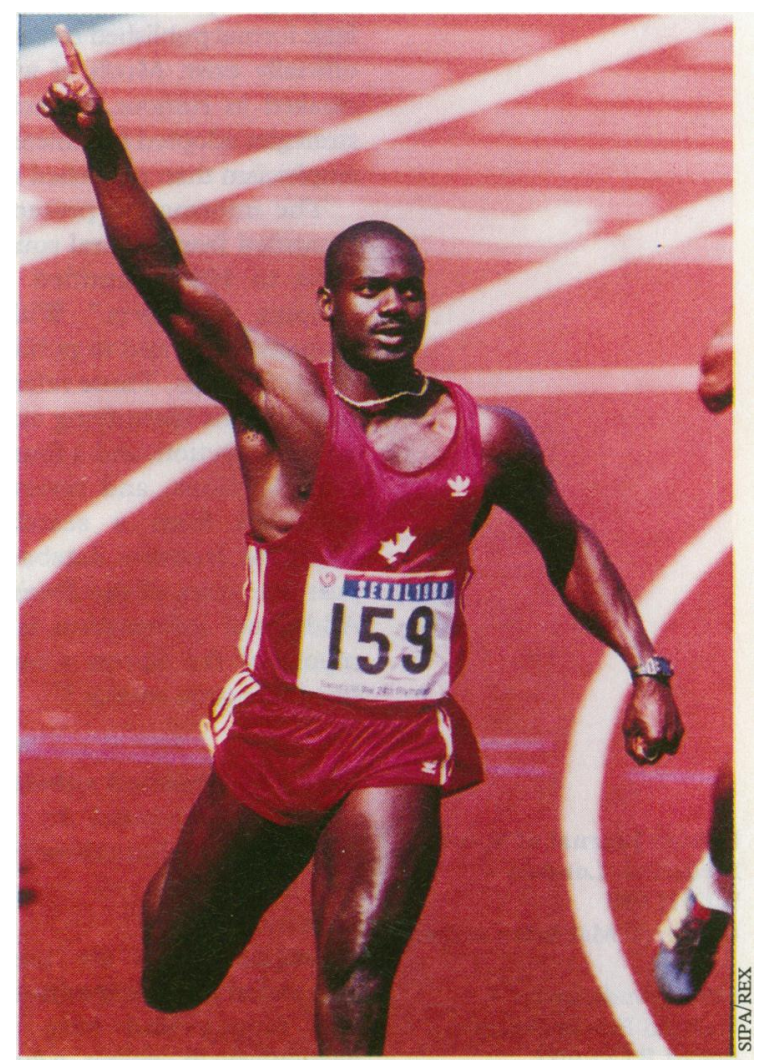

It is almost inevitable that top level athletes may seek advantage through drugs 
Table 1-Results of dope testing (drug control centre, King's College, London)

\begin{tabular}{cccc}
\hline Year & No of tests & $\begin{array}{c}\text { No of } \\
\text { positive } \\
\text { tests }\end{array}$ & $\begin{array}{c}\text { No of } \\
\text { refusals }\end{array}$ \\
\hline 1992 & 4167 & 48 & 10 \\
1993 & 3946 & 41 & 6 \\
1994 & 4374 & 67 & 7 \\
1995 & 4596 & 84 & 15 \\
\hline
\end{tabular}

Data from annual report of Sports Council, 1995-6. ${ }^{1}$

\section{The problems}

We can immediately identify the two main problems of drug control in sport. Some athletes deliberately take performance enhancing drugs and set out to avoid being caught. Others may inadvertently take a substance, even some without performance enhancing effect, and face censure because of a positive drug test result. These factors have major implications for athletes, doctors, and the organisation of the drug testing procedure.

Drug testing, or dope testing, is performed in almost all sports, and sports bodies faced with policing drug abuse publish lists of banned substances. These are substances believed to give the athlete some advantage, though not all prohibited drugs have a proved ergogenic effect. The list is huge and cannot be comprehensive, as many only slightly dissimilar drugs can have similar effect. The only way athletes can remain completely certain that they have not taken a prohibited substance is either to avoid all drugs or to take only those agents on the permitted list. This is not always easy.

Drugs available both on prescription and "over the counter" to treat common conditions such as catarrh or nasal congestion, and even some apparently innocuous preparations for pain relief, may contain banned substances. In addition, athletes who take health food supplements or vitamin products may inadvertently take prohibited substances, especially if these are purchased abroad, as labelling and lists of contents may be imprecise. For example, contents may not be listed on the package, the athlete may not recognise a banned substance, or the name may be colloquial, which may be misleading. Commonly available "over the counter" preparations that do contain a prohibited substance include products such as Bronchipax, Contac 400, Mucron, Nirolex expectorant, and Procol. These are only examples and many other drugs contain substances prohibited in sport.

\section{Information sources for doctors and athletes}

The Sports Council offers an information system so that athletes may find out about various agents and help protect themselves against the inadvertent use of a banned substance. Several booklets are available direct from the Sports Council. In addition, there is a hotline number (0171 3832244$)$ that athletes can use if they require a rapid response. At present this number is linked to an answering machine and the call is returned later. Plans are in hand to offer a direct access line. Even then it will be difficult to supply completely up to date and accurate information. Different sports may have different regulations and while some substances may be banned by one organisation they may be permitted by another. Athletes may be given advice but ultimately may be referred back to their own organisation, as providing a comprehensive list of banned substances for every sport is difficult.

\section{How great is the problem?}

We do not know the magnitude of the problem. We do know, however, the number of positive test results detected by the Sports Council's London testing laboratory (table 1) and note the rising numbers. Data are also available from the games of the 23rd Olympiad in Los Angeles, ${ }^{15}$ where $1.7 \%$ of 1510 samples contained a banned drug. In 1986 at the 10th Asian games $3.2 \%$ of samples were found to contain a banned drug, ${ }^{16}$ and at the 1988 winter Olympics $2.6 \%$ of test results were positive. ${ }^{17}$ We also have data from dope testing in South Africa, ${ }^{18}$ where during 1986-91, 5.5\% of 2066 urine specimens collected from competitors contained drugs classified as forbidden by the International Olympic Committee.

The problem seems to be greatest among body builders. In West Glamorgan Perry et al looked at anabolic steroid use in private gymnasiums and found that $38.8 \%$ of respondents admitted to having taken steroids. ${ }^{19}$ In the west of Scotland $19.5 \%$ of body builders had used drugs to enhance their physique and performance. ${ }^{20}$ In unannounced doping control among body builders in Flanders during 1988-93 between 38\% and $58 \%$ of those tested were found to be positive during this period, ${ }^{21}$ and in a study from the United States over half of the male body builders $(54 \%)$ were using steroids regularly as compared with $10 \%$ of the female competitors. ${ }^{22}$

Even more worrying are figures from the United States, where the prevalence of steroid use among adolescents in large surveys has been found to be between $3.0 \%$ and $7.6 \% .{ }^{23-26}$ Even human growth hormone abuse seems to be a problem in highschools, where $5 \%$ $(n=11)$ of boys reported past or present use and $31 \%$ reported knowing someone who was using growth hormone. $^{27}$

\section{What do athletes think?}

In February 1995 the Sports Council surveyed senior competitors from 26 winter and summer sports. ${ }^{28}$ There was a $60 \%$ response rate (468). Though $74 \%$ had been tested at some point in their career and $66 \%$ thought it likely that they would be tested in the next 12 months, $34 \%$ expressed dissatisfaction at the range of competitors selected for testing and $41 \%$ expressed dissatisfaction about the frequency of testing. Many thought that testing should be more widespread and more often. About $\mathbf{7 0} \%$ believed that testing served as a deterrent, but a quarter believed that lack of widespread testing made the process less of a deterrent for some.

In a study of 1015 Italian athletes over $10 \%$ indicated a frequent use of amphetamines or anabolic steroids at national or international level, fewer athletes mentioning blood doping $(7 \%)$ and $\beta$ blockers $(2 \%)$ or other classes of drugs. ${ }^{29}$ According to over $70 \%$ of athletes,

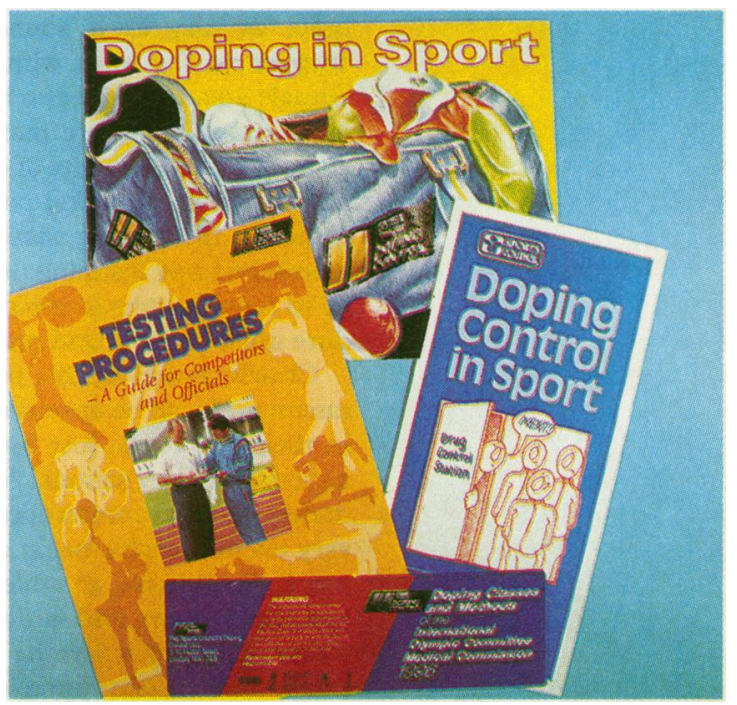

Helpful booklets available from the Sports Council 
access to illegal substances was not difficult. Eighty two per cent wanted stricter controls not only during competitions but also during training.

\section{Drug testing procedure}

The drug testing procedure is as easy as taking a urine sample. This sounds deceptively simple, as testing is a formal and highly regulated procedure to ensure that the urine sample which arrives at the laboratory actually comes from the athlete in question, with no opportunity to tamper with the sample. When selected for testing the athlete is notified by an official and asked to sign a form acknowledging this notification. The athlete, who may be accompanied by an official and must be accompanied if under 16 , attends the testing station within a stipulated period. The testing station should be a private, comfortable place where plenty of drinks are available; often it may be situated in a specially designed mobile testing caravan. Testing is carried out by independent sampling officers, trained and appointed by the Sports Council. Each carries a time limited identity card and a letter of authority for the event to which he or she is allocated.

Before giving a sample the athlete is invited to choose a set of two numbered bottles. Having given the sample (about $100 \mathrm{ml}$ ) the athlete completes a form on a voluntary basis declaring any drug treatment taken in the previous seven days and must check and sign that the sample has been taken and placed in the bottles correctly. The sample is then sent for analysis to a laboratory currently accredited by the International Olympic Committee. In the event of a positive test result the laboratory will notify the governing body of the sport, who will then notify the athlete. What happens then depends on the rules of the governing body of the particular sport. An athlete is usually suspended while a positive result is investigated but has the right to have a second analysis of the urine sample. This analysis may be observed by the athlete himself or herself or by the athlete's representative. There follows a hearing, at which the athlete has an opportunity to present his or her case. It is also possible to appeal, and there have been successful appeals both in the United Kingdom and in the United States.

The testing procedure is of necessity rigorous. Because drugs are potentially such a boost to performance athletes who use them will go to great lengths not to be caught. Thus not only are various agents banned but other manipulative procedures are also banned. These include both mechanical and chemical methods. To ensure that the sample actually comes from the athlete the testing officer must be able to see the urine issue from the meatus and pass into the bottle. This is not quite as easy as it sounds. Male athletes must be stripped to the waist with their shorts to their knees. Female athletes must also be observed very closely during testing. There are many legends of athletes using elaborate arrangements of catheters to provide an alternative sample, bringing condoms filled with drug free urine to the testing station, and even catheterising themselves and instilling drug free urine. If athletes will go to these lengths to avoid detection the testing procedure must be strict and must be enforced.

Clearly, however, this procedure may cause embarrassment. It can be upsetting for young athletes, perhaps in their late teens, just breaking through into the top national or international level, to give a urine sample under these circumstances. Indeed, many people are very uncomfortable being observed giving a urine sample. If, in addition, an athlete has been competing in an endurance sport and is dehydrated or competing at a weight category where he or she is reluctant to drink excess fluid we can appreciate how daunting it can be. It is equally important that athletes should ensure that the testing procedure is observed rigorously for their own protection, that their samples are dispatched in the correct containers, and that all the paperwork is completed with no chance of error. Once a sample is taken there must be a completely secure passage until it arrives in the laboratory.

Elite athletes are subject to year round random testing. Thus an independent sampling officer may call unannounced at any time and request a drug sample. Apparently quite straightforward, there may be organisational difficulties. Many of the most successful athletes travel the world freely and spend periods at warm weather training camps or at altitude. Sometimes finding the athlete can be difficult, and with all the necessary preliminary inquiries it is unlikely that the testing will remain a surprise.

\section{Conclusion}

The only entirely safe way for an athlete to avoid prohibited substances is not to take drugs. There is, however, an overwhelming belief that drugs enhance performance and an athlete may believe that some drug must be taken to level the playing field; hence performance enhancing drugs have been described as coercive drugs. ${ }^{30}$

Can drug taking be stopped? The inevitable answer is that it cannot and that some athletes will always try to seek some extra competitive advantage. Drug taking can be controlled only if detection is likely and the penalties of detection are a sufficient deterrent. Unless there is widespread testing, both in and out of competition, the risk to benefit ratio favours the drug taker. There are therefore considerable difficulties in preventing the use of performance enhancing drugs. There should also, however, be protection for the athlete who inadvertently takes a prohibited substance, and because of the huge implications of a positive test result great care must be taken to avoid having false positive results.

Drug testing is unpleasant but seems to be here to stay. There will always be rumours of undetectable drugs, masking agents, or surgical procedures to subvert the dope test. And always there is the fear that the testers are one step behind and will never quite catch up.

I thank Michele Verroken, of the Sports Council Doping Control Unit; Stephen Martin, of the Sports Council for Northern Ireland; Yasmin Hossain, at the National Sports Medicine Institute of the United Kingdom; and the librarians at the British Medical Association for their help in the preparation of this paper.

1 Sports Council Doping Control Service. Report 1995-96. London: Sports Council, 1996

2 Sports Council Doping Control Unit. Anabolic steroids. London: Sports Council, 1993. (Information booklet No 1.)

3 Sports Council Doping Control Unit. International Olympic Commitree doping classes. London: Sports Council, 1994. (Information booklet No 2.)

4 Sports Council Doping Control Unit. International Olympic Committee accredited laboratories. London: Sports Council, 1994. (Information booklet No 3.)

5 Sports Council Doping Control Unit. Guide to allowable medications. London: Sports Council, 1995. (Information booklet No 4.)

6 Sports Council Doping Control Unit. Suggested further reading. London: Sports Council, 1994. (Information booklet No 5.)

7 Sports Council Doping Control Unit. UK legislation on doping substances. London: Sports Council, 1993. (Information booklet No 6.)

London: Sports Council, 1993. (Information booklet No 6.) Nutrition 1995;5(suppl):S84-99.
.

9 Elashoff JD, Jacknow AD, Shain SG, Braunstein GD. Effects of anabolic-androgenic steroids on muscular strength. Ann Intern Med 1991;115:387-93.

10 Bhasin S, Storer TW, Berman N, Callegari C, Clevenger B, Phillips J, et al. The effects of supraphysiologic doses of testosterone on muscle size and strength in normal men. $N$ Engl 9 Med 1996;335:1-7.

11 Raynaud E, Audran M, Brun JF, Fedou C, Chanal JL, Orsetti A. False-positive cases in detection of testosterone doping. Lancet 1992;340:1468-9.

12 Kicman AT, Cowan DA, Myhre L, Nilsson S, Tomten S, Oftebro H. Effect on sports drug tests of ingesting meat from steroid- (methenolone)treated livestock. Clin Chem 1994;40:2084-7.

13 Kruse P, Ladefoged J, Nielsen, Paulev PE, Sorensen JP. Beta-blockade used in precision sports: effects on pistol shooting performance. 9 Appl Physiol in precision sport: 
14 Berglund B, Hemmingson P. Effect of reinfusion of autologous blood on exercise performance in cross-country skiers. Int $f$ Sports Med 1987;8:231-3.

15 Catlin DH, Kammerer RC, Hatton CK, Sekera MH, Merdink JL. Analytical chemistry at the games of the XXIIIrd Olympiad in Los Angeles, 1984. Clin Chem 1987:33:319-27.

16 Park J. Doping test report of 10th Asian games in Seoul. f Sports Med Phys Fitmess 1991;31:303-17.

17 Chan SC, Torok-Both GA, Billay DM, Przybylski PS, Gradeen CY, Pap $\mathrm{KM}$, et al. Drug analysis at the 1988 Olympic Winter Games in Calgary. Clin Chem 1991;37:1289-96.

18 van der Merwe PJ, Kruger HS. Drugs in sport-results of the past 6 years of dope testing. South Afr Med F 1992;82:151-3.

19 Perry HM, Wright D, Littlepage BN. Dying to be big: a review of anabolic steroid use. Br ₹ Sports Med 1992;26:259-61.

20 McKillop G. Drug abuse in body builders in the west of Scotland. Scott Med f 1987;32:39-41

21 Delbeke FT, Desmet N, Debackere $M$. The abuse of doping agents in competing body builders in Flanders (1988-1993). Int I Sports Med 1995;16:66-70.
22 Tricker $\mathrm{R}$, O'Neill MR, Cook $\mathrm{D}$. The incidence of anabolic steroid use among competitive bodybuilders. I Drug Educ 1989;19:313-25.

23 Whitehead R, Chillag S, Elliott D. Anabolic steroid use among adolescents in a rural state. 9 Fam Pract 1992;35:401-5.

24 Komoroski EM, Rickert VI. Adolescent body image and attitudes to anabolic steroid use. Am $\mathcal{Y}$ Dis Child 1992;146:823-8.

25 Terney R, McLain LG. The use of anabolic steroids in high school students. Am f Dis Child 1990;144:99-103.

26 Windsor R, Dumitru D. Prevalence of anabolic steroid use by male and female adolescents. Med Sci Sports Exerc 1989;21:494-7.

27 Rickert VI, Pawlak-Morello C, Sheppard V, Jay MS. Human growth hormone: a new substance of abuse among adolescents? Clin Pediatr 1992;31:723-6.

28 Sports Council. Doping control in the UK. A survey of the experiences and views of elite competitors. London: Sports Council, 1995.

29 Scarpino V, Arrigo A, Benzi G, Garattini S, La Vecchia C, Bernardi LR, et al. Evaluation of prevalence of "doping" among Italian athletes. Lancet 1990;336:1048-50.

30 Lombardo J. Drug control programmes. Br $f$ Sports Med 1996;30:82-3.

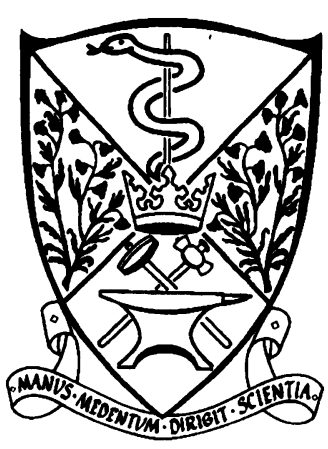

Department of Gastroenterology, Hammersmith Hospital, London W12 0HS Case presented by: Farah B Ahmed, senior house officer in gastroenterology Chairman: J Scott, director of medicine

Discussion group:

H J F Hodgson, professor of gastroenterology C M Oakley, professor of cardiology

J S Friedland, consultant in infectious diseases

$\mathbf{P}$ Ind, consultant in

respiratory medicine

Histopathology review:

G Stamp, consultant in

histopathology

Series editor: Dr S D

Taylor-Robinson.

BMF 1996;313:215-7

\section{Grand Rounds-Hammersmith Hospital}

\section{Tuberculous enteritis}

\section{$A$ serious possibility in some patients}

Abdominal tuberculosis should always be considered in immigrants from regions where this disease is endemic who present with abdominal signs and symptoms. We describe the case of a young man from such a region with extensive tuberculous involvement of his gastrointestinal tract and peritoneum. This case highlights the difficulties in diagnosing tuberculous enteritis and the need to consider seriously the possibility of this disease in such patients.

Tuberculous enteritis remains a challenge to the diagnostic acumen and therapeutic skills of both the physician and the surgeon.

\section{Case history}

A 25 year old Eritrean student, resident in Britain since 1989, first sought medical advice at this hospital in April 1994, when he was being investigated in relation to emigration to Canada. Plain chest radiography at this time was initially reported as showing a pulmonary nodule. On review, however, it was considered to be normal.

He presented a year later to the gastroenterology department with large amount of weight loss $(20 \mathrm{~kg}$ over a year), acid reflux, epigastric pain, intermitten vomiting, occasional bloody stools, and depression. $\mathrm{He}$ had no history of illicit substance misuse and no risk factors for HIV infection. He admitted having a very erratic eating pattern with poor nutritional intake. $\mathrm{He}$ was a non-smoker and did not drink alcohol.

On examination he was extremely cachectic, weighing only $49 \mathrm{~kg}$. He had a left sided proptosis and leukonychia, and he was clinically anaemic. Ascites was also present, but there was no lymphadenopathy, peripheral oedema, or chronic liver disease.

Investigations showed a hypochromic, normocytic anaemia (haemoglobin concentration $99 \mathrm{~g} / 1$, mean corpuscular volume $84 \mathrm{fl}$ ), a low serum iron concentration $(1.6 \mu \mathrm{mol} / \mathrm{l})$, low transferrin concentration $(0.85 \mathrm{~g} / \mathrm{l})$, low transferrin saturation index $(7 \%)$, and raised ferritin concentration $(367 \mu \mathrm{g} / \mathrm{l})$. He had raised inflammatory markers ( $\mathrm{C}$ reactive protein concentration $\mathbf{7 7}$ $\mathrm{U} / \mathrm{l}$, erythrocyte sedimentation rate $65 \mathrm{~mm}$ in the first hour) and a low serum albumin concentration ( $12 \mathrm{~g} / 1)$. His total white cell count and remaining biochemical profile were normal. Blood and stool cultures were sterile and negative for acid fast bacilli. Chest radiography was unremarkable as were sigmoidoscopy, colonoscopy, and biopsies. Oesophagogastroduodenoscopy showed

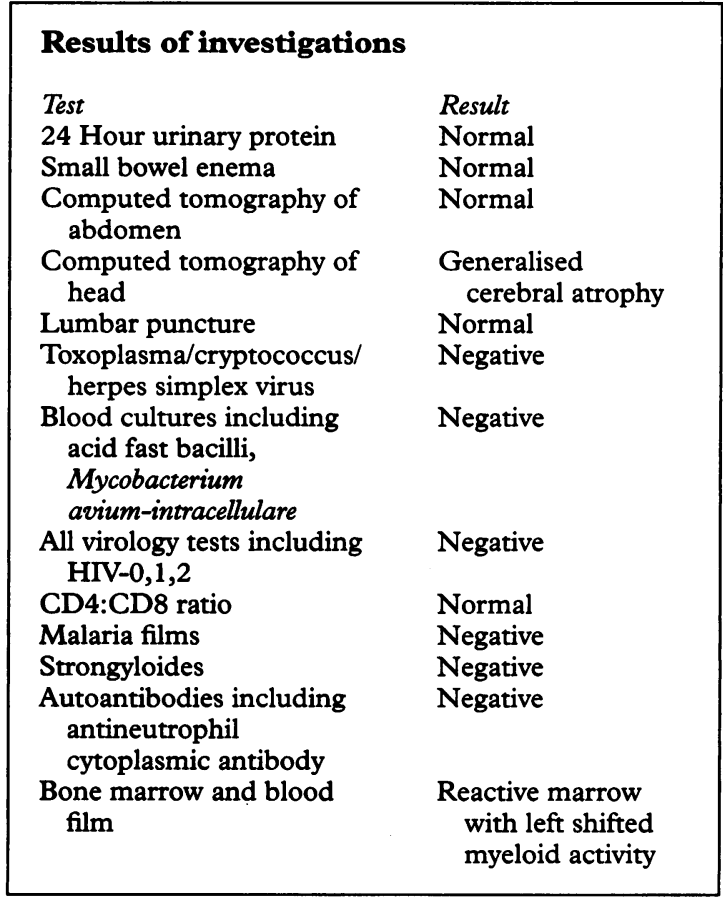

oesophageal candidiasis, a benign gastric ulcer, mild duodenitis, and Helicobacter pylori infection.

$\mathrm{He}$ was admitted for further investigations and treated with omeprazole, metronidazole, and amoxycillin for $H$ pylori. He became febrile and expressed paranoid delusions and increasing confusion. He was started on multivitamins and thiamine in view of his malnourished state. The working diagnosis was a chronic infectious aetiology, retroviral or tuberculous, with a broad differential of small bowel pathology or a protein-losing enteropathy. However, no acid fast bacilli were seen in either cerebrospinal fluid or peripheral blood, and no organisms were grown with standard culture techniques. Despite numerous investigations, including HIV tests, no positive results were obtained (box).

Five days after admission he had a grand mal seizure and was started on phenytoin. A contrast enhanced computed tomogram of the head showed generalised cerebral atrophy with prominent ventricles and subarachnoid spaces; magnetic resonance imaging done before and after gadolinium enhancement suggested 